\title{
Sequence and expression of the Mycobacterium leprae dnaJ gene
}

\author{
Suzanna S. Harvey, ${ }^{1}$ Kirsten R. McKenzie, ${ }^{1}$ Paul W. Roche ${ }^{1}$ and Warwick J. Britton ${ }^{1,2 *}$ \\ ${ }^{1}$ Centenary Institute of Cancer Medicine and Cell Biology and ${ }^{2}$ Department of Medicine, University of Sydney, \\ NSW 2006, Australia
}

(Received 10 August 1992; revised 26 March 1993; accepted 19 April 1993)

\begin{abstract}
Study of Mycobacterium leprae, the causative agent of leprosy, has been advanced by the isolation of genes encoding mycobacterial proteins including $d n a K$ encoding the $M$. leprae $70 \mathrm{kDa}$ heat shock protein. The sequence downstream from $d n a K$ revealed a second open reading frame coding for a protein of 389 amino acids with a calculated molecular mass of $41 \cdot 2 \mathrm{kDa}$. Sequence analysis demonstrated significant DNA homology with the dnaJ gene of other organisms. High amino acid sequence identity was obtained between the DnaJ protein of $M$. leprae and $M$. tuberculosis ( $89 \%$ ) with significant divergence between the two occurring only at the C-terminal end. The expressed recombinant DnaJ protein had a molecular mass of $42 \mathrm{kDa}$.
\end{abstract}

\section{Introduction}

Mycobacterium leprae, which is a significant human pathogen, cannot be cultivated outside an animal host. This has provided difficulties for the biochemical and immunological study of $M$. leprae proteins. The molecular cloning of $M$. leprae DNA has offered an alternative method for the characterization and production of $M$. leprae proteins (Young et al., 1985).

The gene encoding a significant $M$. leprae antigen, the $70 \mathrm{kDa}$ heat shock protein and DnaK homologue (p70), was cloned by identification of the gene product with a mycobacterial specific mouse monoclonal antibody $\mathbf{L} 7$ (Britton et al., 1985; McKenzie et al., 1991). In Escherichia coli, the $d n a K$ heat shock promoter controls an operon containing the $d n a K$ gene and a second heatshock gene, dnaJ (Cowing et al., 1985). Initial sequencing downstream of the $M$. leprae p70 gene revealed an open reading frame, the translational product of which demonstrates some homology at the amino acid level with the dnaJ gene of E. coli (Garsia, 1989). Alignment of the $M$. leprae and $E$. coli sequences indicated that the $M$. leprae dnaJ gene begins $700 \mathrm{bp} \mathrm{3'}$ to the dnaK TGA stop codon. Recent analysis of the dnaK-grpE-dnaJ gene cluster of M. tuberculosis demon-

*Author for correspondence. Tel. 25167098 ; fax 26923968.

Abbreviation: GST, glutathione $S$-transferase.

The nucleotide sequence data reported in this paper have been submitted to GenBank and have been assigned the accession number M95576. strated that the $M$. tuberculosis dnaJ gene begins 792 bp downstream of the dnaK (Young et al., 1988; Lathigra et al., 1988; D. Young, personal communication). We have now isolated the complete $M$. leprae dnaJ gene and in this report present its DNA sequence and the expression of the recombinant $M$. leprae DnaJ protein.

\section{Methods}

DNA sequence analysis. A $3.6 \mathrm{~kb}$ Eco RI fragment of the cosmid clone ML231 (McKenzie et al., 1991) was subcloned into the plasmid vector pBluescript SK and designated pSKdnaJ. The $3.6 \mathrm{~kb}$ fragment contained the $3^{\prime}$ part of the $M$. leprae dnaK gene, previously reported as the fragment JKL2 (Garsia et al., 1989; McKenzie et al., 1991), and an additional $1.5 \mathrm{~kb}$ of $M$. leprae DNA. pSKdnaJ was denatured (Zhang et al., 1988) prior to sequencing with the Sequenase version 2.0 DNA sequencing kit (United States Biochemical Corp.) according to the manufacturer's instructions. Synthetic oligonucleotides designed using the DNA sequence downstream of M. leprae dnaK (Garsia, 1989) served as primers. Both strands of DNA were sequenced and the amino acid sequence of the open reading frame deduced. Homology searches used the MATChFasta programs of Pearson \& Lipman (1988) and sequence comparisons were made using the GCG package (Genetics Computer Group Inc., Madison, WI, USA). The databases were accessed via ANGIS (Australian National Genomic Information Service, University of Sydney).

Expression of recombinant $M$. leprae dnaJ. The $M$. leprae dnaJ gene was ligated to the $3^{\prime}$ end of the coding sequence for the C-terminal portion of the glutathione $S$-transferase (GST) of Schistosoma japonicum, under the control of the lac $Z$ promoter, in the expression vector pGEX-2T (Smith \& Johnson, 1988). A $1.5 \mathrm{~kb}$ fragment of DNA was amplified from plasmid pSKdnaJ by polymerase chain reaction (PCR) (Higuchi et al., 1988). An oligonucleotide complementary to the $5^{\prime}$ end of the $M$. leprae dnaJ gene and the pBluescript reverse sequencing primer were used as primers in the PCR reaction. The PCR DNA product, containing a unique $3^{\prime} E c o$ RI site derived from pBluescript, was digested with EcoRl and ligated between the SmaI 
TGACCGAAGGCAATCCACACGAACAGGTGACGGTCACCGACAAACGACGGATCGATCCCGATACTGGCGAAGTACGATCT

ATACCTCTCGCGACACGCCGGGAGGTAGCGAGCCGGCTGGTATGACCGAAGTGCCAACAGMACCGACACAATGGTCCGAC

160

AAPGTTGCTGAGCTGACTAGCGATCTGCAGCGGGTGCAGECCGATTTGCCAACTACCGTAAGCGAGCGTTGCGCGACCA

240

GCAGACGGCTTCGGATCGAGCCAAGGCGAGTGTCATCAGCCAATTGTTGGGTGTGCTTGACGATTTCGATCGTGCCCGCG

320

AGCACGGCGATCTGGACTCGGGTCCGCTGAAGTCGGTCGCCGACAAGCTAAGAGCGCATIGACCGGGCTCGGGCTGOTGG

400

CGTTTGGCGTCGAGGGAGAAGACTTCGACCCGGTGCTGCACGAAGCCGTGCAGCATGAGGGCGATGGTGGGGAAGGCTCC

480

AAGCCGGTGATCGGTGACGTCCTCCGGCACGGCTACAAATTGGGCGATCAAGTGCTGCGGCACGCCTTGGTCGGGGTGGT

560

TGACACGATCGCAGGCGATGGIGCCGAAACGGTTCCATAGTCGCACCTGTCGACAGCACAGCCAAAACCGAGCAAGGTG

'S-D'

AACTGGGCGATAADGTGACACCGCACAAGGAGGACGGTGGTAGGGGGTCACGCGAT ATG GCC CAG CAT GAA TGG

met ala gln arg glu trp

640

714

6

GTC GAA AAG GAC TTC TAC AAG GAG CTA GGC GTT TCC TCT GAC GCC AGT CCC GAA GAG ATT val glu lys asp phe tyr lys glu leu gly val ser ser asp ala ser pro glu glu tle

AAC CGT GCC TAT CGG AAG TI'G GCA CGC TAC CTI CAT CCT GAC GCT AAT CCC GAC AAC TCC asn arg ala tyr arg lys leu ala arg tyr leu his pro asp ala asn pro ala asn ser

TCP GGT GAA CGG TTT AAG GTG GTG TCT GAG GCG CAC AAT GTG TTG TCG GAT CCG GIC AAG ser gly glu arg phe lys val val sex glu ala his asn val leu ser asp pro val lys

CGC AAA GAG TAC GAT GAA ACT CGC CGG CTG TTT GCC GGC GGC TGC TTA GGC GGT CGT CGG arg lys glu tyr asp glu thr arg arg leu phe ala gly gly cys leu gly gly arg arg

TTC GAC AGT AGT TTT GGT GAC TTT GGA ATG GGC GGC GAC GGT GCT GAG TTC AAC CTC AAC phe asp ser ser phe gly asp phe gly met gly gly asp gly ala glu phe asn leu asn

GAC TTG TTC GAT GCT GCC AGT CGA ACC GGG GGC ACC AAC ATC GGT GAC TTG TTC GGT GGC asp leu phe asp ala ala ser arg thr gly gly thr asn ile gly asp leu phe gly giy

1014

106

1079

126

TTG TIT GGA CGC GGC GCC AGT GTC CGC CCC AGT AGC CGA CGC GGC AAC GAC CTG GAG ACC leu phe gly arg gly ala ser val arg pro ser ser arg arg gly asn asp leu glu thr

GAG GCA GAT TTG GAT TTT GTC GAA GCG GCC AAA GGT GTA GCG ATG CCG CTG CGG CTG ACC glu ala asp leu asp phe val glu ala ala lys gly val ala met pro leu arg leu thr

1194

166

AGC CCA GCG CCG TGC ACC AAT TGC CAT GGC AGC GGG GCA CGT CCG GGA ATG AGT CCG AAA 1254 ser pro ala pro cys thr asn cys his gly ser gly ala arg pro gly ile ser pro iys

186

GTG TGT TCC ACT TGC AAC GGT TCC GGC GTG ATC AAC CGC AAT CAG GGT GCC TTT GGT TTC 1314 val cys ser thr cys asn gly ser gly val ile asn arg asn gln gly ala phe gly phe 206

TCT GAG CCT TGT ACC GAA TGT CGG GGT AGC GGG TCG ATC ATC GAG CAC CTC CGC GAA GAG 1374 ser glu pro cys thr glu cys arg gly ser gly ser ile ile glu his leu arg glu glu 226

TGC AAA GGC ACC GGG GTT ACC ACC CGG ACC CGA ACC ATC AAT GTG CGG ATC CCG TCC GGT 1434 cys lys gly thr gly val thr thr arg thr arg thr ile asn val arg ile pro ser gly 246

GTC GAA GAT GGA CAA CGG ATT CGG CTG GCA GGG CAG GGC GAG GCG GGG TTG CGT GGC GCT 1494 val glu asp gly gln arg lle arg leu ala gly gln gly glu ala glu leu arg gly ala 266

CCG TCG GGT GAT CTG TAT GTG ACG GTC CAT GTG CGG CCT GAC AAG ATC ITT GGT CGA AAT 1554 pro ser gly asp leu tyr val thr val his val arg pro asp lys ile phe gly arg asn 286

GGC GAC GAC CTC ACC GTG ACC ATT CCG GTT AGC TTC ACT GAA TTA GCT TTG GGC TCA ACC 1614 gly asp asp leu thr val thr ile pro val ser phe thr giu ieu ala leu gly ser thr 305 


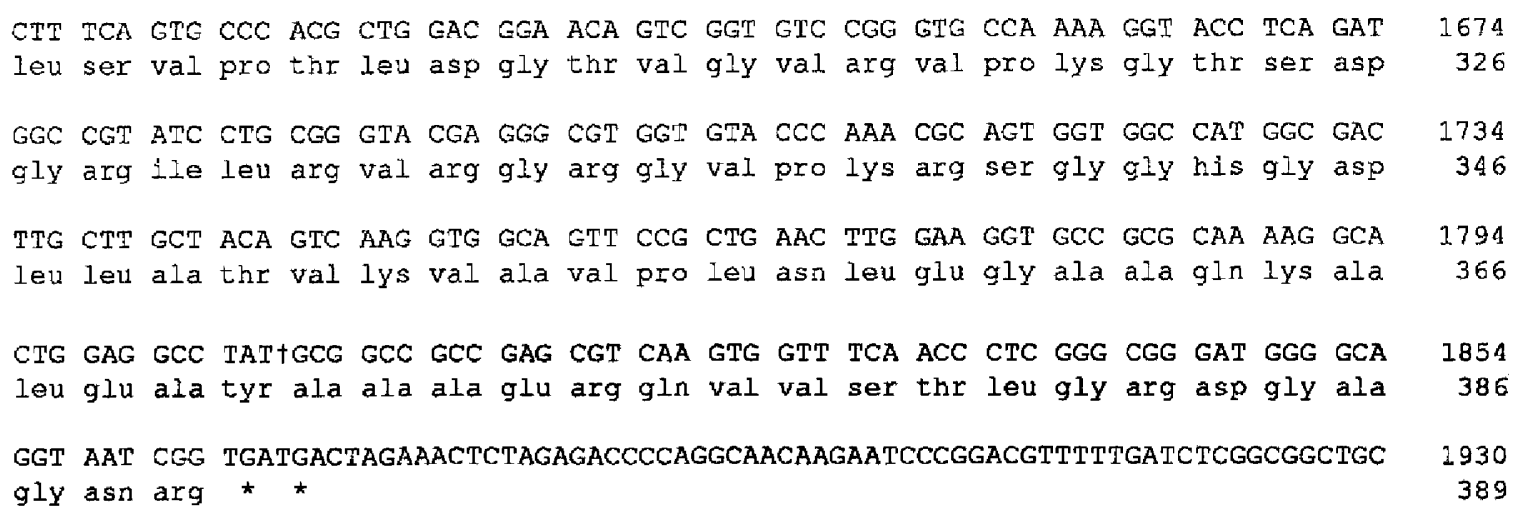

Fig. 1. Nucleotide and deduced amino acid sequence of the $M$. leprae dna $J$ gene. Potential promotor sequences are boxed. A Shine-Dalgarno (S-D) sequence is underlined; * represents a stop codon. The numbering of nucleotides begins with the stop codon of the $d$ na $K$ open reading frame and numbering of amino acids is from the start of the coding region. Amino acids are represented by their three letter abbreviations.

and $E c o$ RI sites of the expression vector pGEX-2T. The presence, in frame, of the $M$. leprae dnaJ gene was confirmed by sequencing from the 3 end of the GST coding sequence within the new construct. Recombinant $M$. leprae DnaJ protein fused to GST (DnaJf) was cleaved with thrombin to yield soluble DnaJ (DnaJc) (Smith \& Johnson, 1988; McKenzie et al., 1991).

Electrophoresis. The recombinant proteins were separated by $12 \%$ SDS-PAGE and transferred to nitrocellulose paper as described (McKenzie et al., 1991). The immunoblots were probed with antisera from Balb/c mice immunized with recombinant cleaved DnaJ in incomplete Freund's adjuvant.

\section{Results and Discussion}

\section{DNA sequence of the M. leprae dnaJ gene}

A total of $1930 \mathrm{bp}$ of DNA sequence was determined downstream of the $M$. leprae dnaK gene. The largest open reading frame within this region begins 700 nucleotides downstream of the $d n a K$ gene and encodes a protein of 389 amino acids with a calculated molecular mass of $41.2 \mathrm{kDa}$ (Fig. 1). Sequence homology of this open reading frame with the sequences of dnaJ from other organisms strongly supported the notion that the sequence encoded a DnaJ equivalent protein in $M$. leprae. A Shine-Dalgarno sequence was identified immediately upstream of the DnaJ coding region, in addition to several possible promotors. Expression of dnaJ may therefore be under the control of its own promotor in $M$. leprae rather than using the same promotor as the dnaK gene, as has been suggested for other bacteria, including $M$. tuberculosis (Dale \& Patki, 1990 ) and E. coli (Cowing et al., 1985).

\section{Expression of the recombinant $M$. leprae DnaJ protein}

The $M$. leprae dnaJ gene was cloned into the expression vector pGEX-2T resulting in the production of recombinant $M$. leprae DnaJ protein fused to GST (Dna.If), with a molecular mass of $66 \mathrm{kDa}$ (Fig. $2 a$ ). The fusion protein reacted strongly with polyclonal rabbit anti-GST antisera (data not shown) and murine anti $M$. leprae DnaJc (Fig. 2b). Cleavage of this fusion protein with thrombin resulted in the release of recombinant $M$. leprae DnaJ protein (DnaJc) with a molecular mass of $42 \mathrm{kDa}$ (Fig. 2). The DnaJc protein was partially degraded by thrombin resulting in additional immunoreactive bands of $32 \mathrm{kDa}$ and less than $15 \mathrm{kDa}$ in the DnaJc sample (Fig. 2).

\section{Comparison of the DnaJ protein sequences}

The amino acid sequence of the $M$. leprae DnaJ protein was compared with those in the GenBank and EMBL data bases. A comparison of the amino acid sequence of $M$. leprae DnaJ with those from the prokaryotes $M$. tuberculosis and $E$. coli and the eukaryotic yeast Saccharomyces cerevisiae is shown in Fig. 3. The maximum amino acid sequence identity $(89 \%)$ was obtained with the DnaJ protein of the closely related $M$. tuberculosis. The $M$. leprae DnaJ protein is 33 amino acids $(9 \%)$ longer than that of $M$. tuberculosis (356 amino acids) and considerable sequence divergence was observed at the C-terminal end, where $80 \%$ of the $15 \mathrm{C}$ terminal residues of $M$. tuberculosis varied from the $M$. leprae sequence. Conservation is highest in the internal two-thirds of DnaJ with $94 \%$ identity occuring between amino acids $97-335$ in the proteins of $M$. leprae and $M$. tuberculosis.

The lower degree of conservation (24-42\% amino acid identity) shown between the DnaJ proteins of mycobacteria and those of other organisms (including $E$. coli, Bacillus subtilis, Caulobacter crescentus, $S$. cerevisiae and Homo sapiens) contrasts with the $45-55 \%$ sequence identity observed between the prokaryotic and eukaryotic 65 and $70 \mathrm{kDa}$ heat shock proteins (McKenzie et al., 
(a)

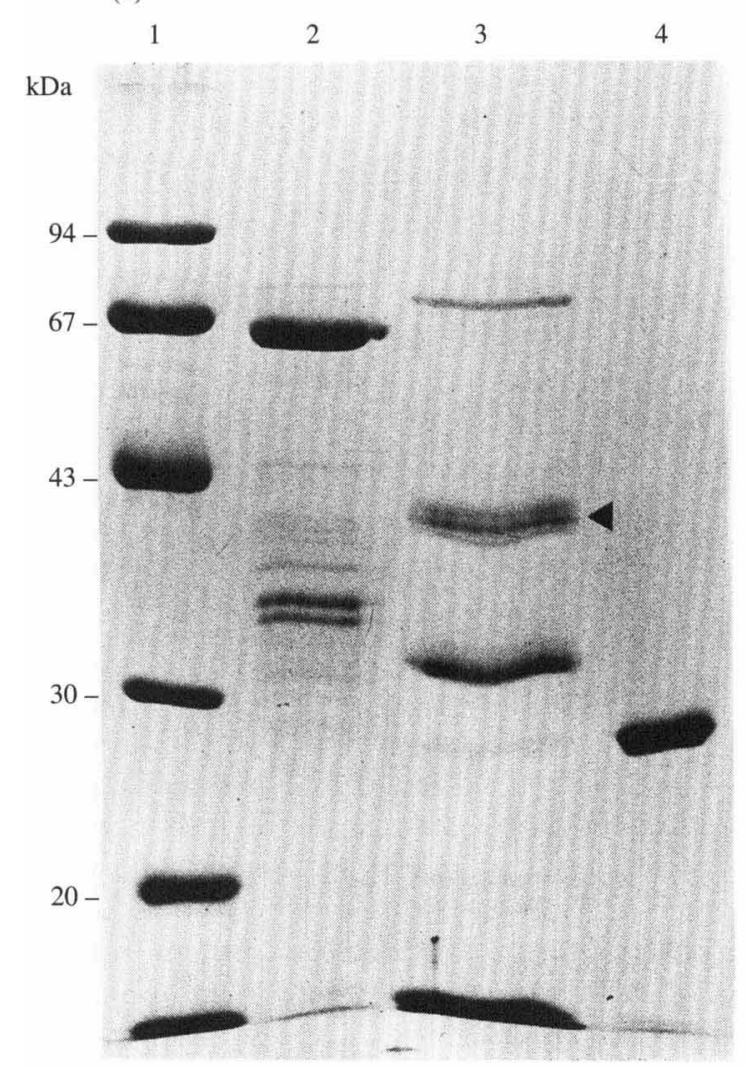

(b)

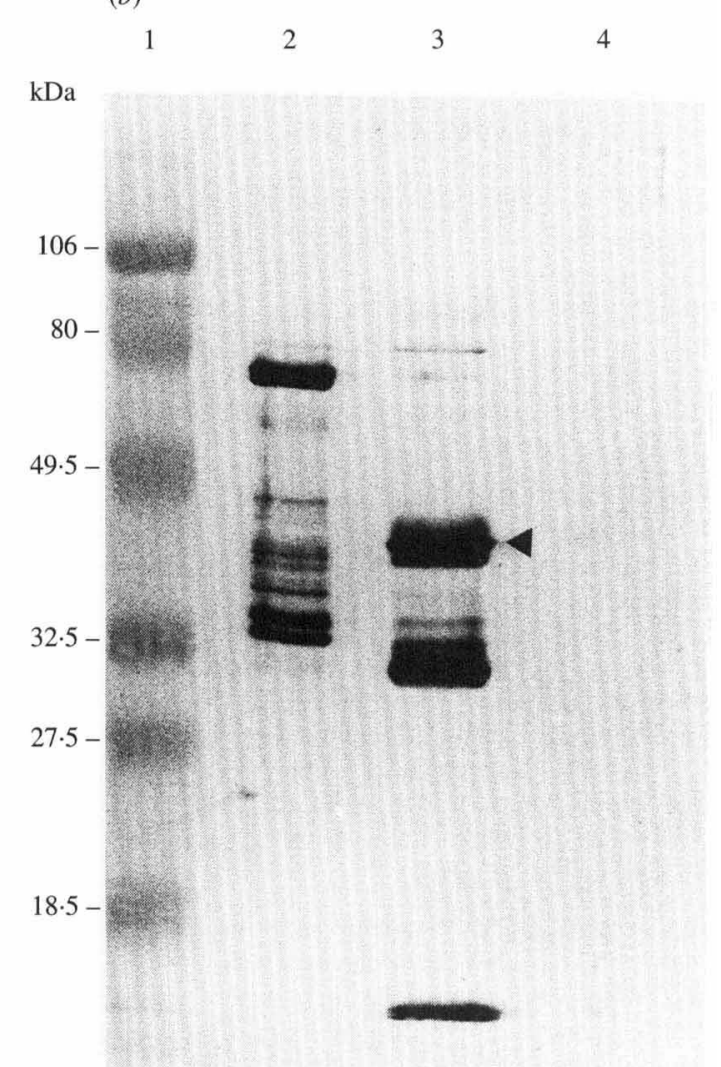

Fig. 2. SDS-PAGE and immunoblot of recombinant $M$. leprae DnaJ. The separated proteins ( $10 \mu \mathrm{g}$ per track) were stained with Coomassie Brilliant Blue $(a)$. The proteins $(2 \mu \mathrm{g}$ per track) were transferred to nitrocellulose and probed with murine anti-DnaJ antiserum (b). Tracks: 1, mol. mass markers; 2, GST-DnaJ fusion protein (DnaJf); 3, DnaJ cleaved protein (DnaJc); 4, GST. The position of the cleaved DnaJc protein is indicated by an arrowhead.

1991; Young et al., 1988). In the case of the E. coli and mycobacterial DnaJ proteins, there are two regions of greater conservation: residues $10-40$ (50\% identity) and $238-277$ ( $68 \%$ identity). In contrast, there is a gap in the E. coli protein corresponding to positions $100-121$ in the $M$. leprae product. The four tandem repeats of a motif consisting of Cys-X-X-Cys-X-Gly-X-Gly identified in M. tuberculosis and E. coli DnaJ proteins (Lathigra et al., 1988) are also conserved in the $M$. leprae DnaJ protein (Fig. 3), except that the fourth copy lacks the initial Cys residue. The conserved regions may be significant in the proposed function of DnaJ in binding to subunits during the assembly of multimeric proteins (Langer et al., 1992). The highly conserved $\mathrm{N}$-terminal region of the amino acid sequence of the related $70 \mathrm{kDa}$ heat shock protein is necessary for the ATPase activity of the molecule (Peake et al., 1991). In contrast, the C-terminal region of Hsp70 varies between species, with the terminal 70 residues of $M$. leprae being completely divergent from those of the M. tuberculosis protein (McKenzie et al., 1991). Divergence of the mycobacterial DnaJ protein from $E$. coli and eukaryotic DnaJ proteins could be explained if the mycobacterial gene products are no longer utilized.
However, this is unlikely as the expression of the $M$. tuberculosis DnaJ protein is increased at higher temperatures (Lathigra et al., 1991). The high level of conservation between the $M$. leprae and $M$. tuberculosis DnaJ proteins would suggest that $M$. leprae DnaJ is also expressed. However, it is not possible to test this experimentally at present.

In $E$. coli, the GrpE heat shock protein has been shown to collaborate with the DnaK and DnaJ proteins in the folding of some proteins (Langer et al., 1992). DnaK recognizes extending polypeptide chains and cooperates with DnaJ in stabilizing this intermediate conformation. The GrpE protein and ATP hydrolysis are required for both the transfer of the protein to GroEL (hsp60) and subsequent GroEL-mediated folding of the native protein. The grpE gene of $M$. tuberculosis resides between the dnaK and $d n a J$ genes. The corresponding region in $M$. leprae shows $78 \%$ nucleotide identity with that of $M$. tuberculosis. However, alignment of the two mycobacterial sequences revealed two gaps in the $M$. leprae sequence of 46 and 40 nucleotides corresponding to regions of the $M$. tuberculosis sequence containing the first 22 nucleotides and the last 34 


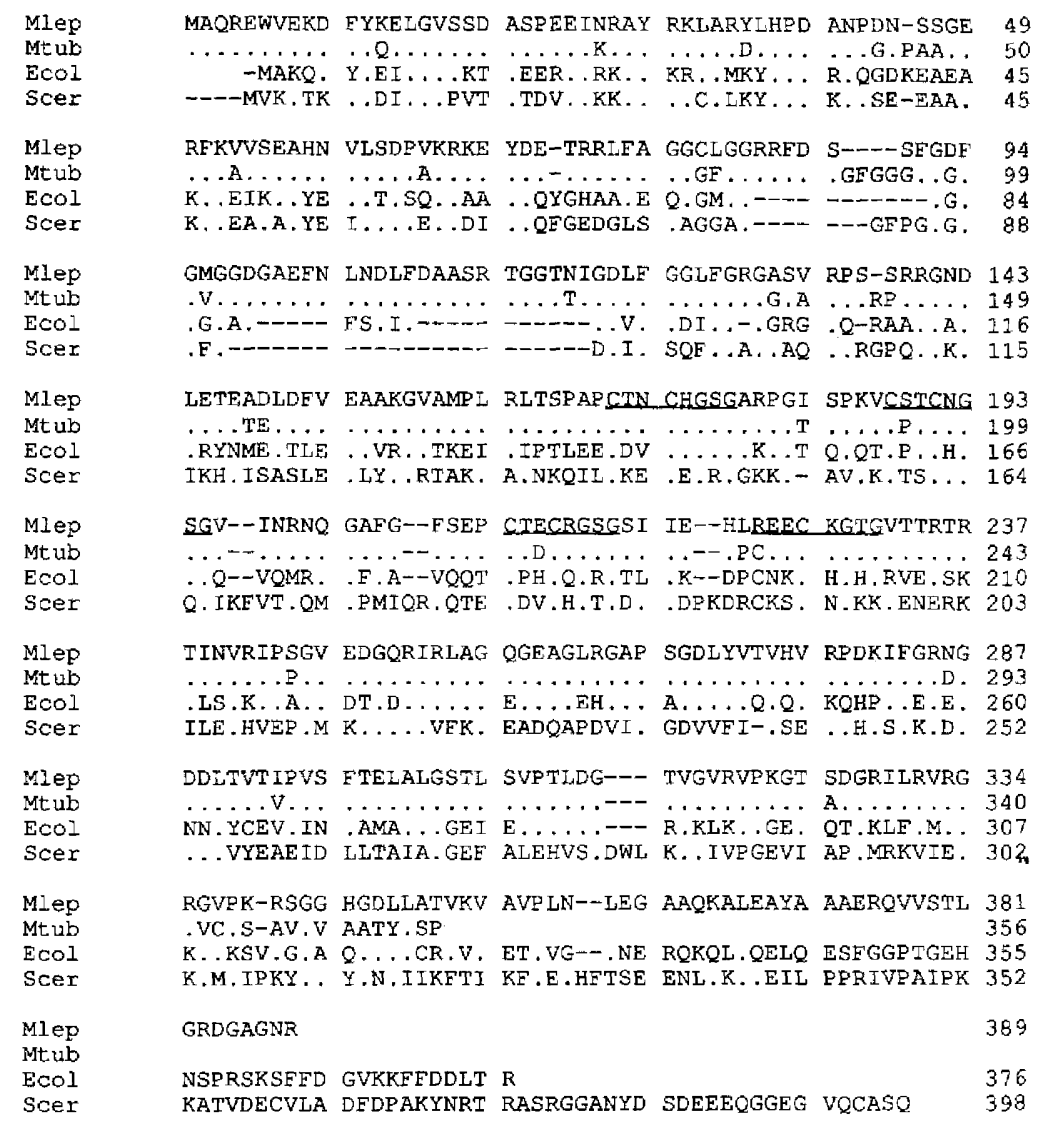

Fig. 3. Alignment of the $M$. leprae DnaJ deduced amino acid sequence with DnaJ amino acid sequences from different species. Mlep, $M$. leprae DnaJ; Mtub, $M$. tuberculosis DnaJ (Lathigra et al., 1988); Ecol, E. coli DnaJ (Bardwell et al., 1986); and Scer, S. cerevisiae DnaJ (Caplan \& Douglas, 1991). Amino acids are represented by their single letter abbreviations. Amino acids identical to those of $M$. leprae are represented as a full stop (.). Gaps in the sequence, where additional amino acids are present in the sequence of other species, are represented as a hyphen(-). The four tandem repeats of the conserved Cys-XX-Cys-X-Gly-X-Gly motif (Lathigra et al,, 1988) are underlined in the $M$. leprae sequence. nucleotides of the grpE gene. There are additional nucleotide differences between the two mycobacterial sequences. Further, there is no significant open reading frame in this region of the $M$. leprae genome. In $B$. subtilis, $\operatorname{grp} E$ is thought to be expressed as part of the dnaK operon (Wetzstein \& Schumann, 1990), whereas in $E$. coli it forms part of a separate operon. The latter, together with the apparent ubiquity of the GrpE protein among prokaryotes suggests that an intact copy of the grpE gene is likely to be present elsewhere in the $M$. leprae genome.

This work was supported by the National Health and Medical Research Council of Australia.

\section{References}

Bardweill, J., Tilly, K., Craig, E., King, J., Zylicz, M. \& Georgopoulos, C. (1986). The nucleotide sequence of the Escherichia coli K-12 dnaJ gene: a gene that encodes a heat shock protein. Journal of Biological Chemistry 261, 1782-1785.

Britton, W. J., Hellqvist, L., Basten, A. \& Raison, R. (1985). Mycobacterium leprae antigens involved in human immune responses. I. Identification of four antigens by monoclonal antibodies. Journal of Immunology 135, 4171-4177.

CAPLAN, A. \& Douglas, M. (1991). Characterization of YDJl : a yeast homologue of the bacterial DnaJ protein. Journal of Cell Biology 114, 609-621.

Cowing, D., Bardwell, J., Craig, E., Woolford, C., Hendrix, R. \&
Gross, C. (1985). Consensus sequence for the Escherichia coli heat shock gene promotors. Proceedings of the National Academy of Sciences of the United States of America 82, 2679-2683.

DALE, J. \& PATKI, A. (1990). Mycobacterial gene expression and regulation. In Molecular Biology of the Mycobacteria, pp. 173-198. Edited by J. McFadden. Guildford, UK: Surrey University Press. Garsia, R. (1989). PhD thesis, University of Sydney, Australia.

Garsia, R., Hellqvist, L., Booth, R., Radford, A., Briston, W., Astbury, L., Trent, R. \& Basten, A. (1989). Homology of the $70 \mathrm{kD}$ antigens from Mycobacterium leprae and $M$. bovis with the $M$. tuberculosis $70 \mathrm{kD}$ antigen and with the conserved heatshock protein 70 of eukaryotes. Infection and Immunity 57, 204-212.

Higlchi, R., KRUmmel, B. \& Scilli, R. (1988). A general method of in vitro preparation and specific mutagenesis of DNA fragments: study of protein and DNA interactions. Nucleic Acids Research 16, 7351.7367.

Langer, T., Lu, C., Echols, H., Flanagan, J., Hayer, M. K. \& HartL, F. U. (1992). Successive action of DnaK, DnaJ and GroEL along the pathway of chaperone-mediated protein folding. Nature, London 356, 683-689.

Lathigra, R., Young, D., Sweetser, D. \& Young, R. (1988). A gene from Mycobacterium tuberculosis which is homologous to the DnaJ heat shock protein of E. coli. Nucleic Acids Research 16, 1636.

Lathigra, R., Butcher, P., Garbe, T. \& Young, D. (1991). Heat shock proteins as virulence factors of pathogens. Current Topics in Microbiology and Immunology 167, 125-145.

McKenzie, K., Adams, E., Britton, W., Garsia, R. \& Basten, A. (1991). Sequence and immunogenicity of the $70 \mathrm{kD}$ heat shock protein of Mycobacterium leprae. Journal of Immunology 147, 312-319.

Peake, P., Basten, A. \& Britron, W. J. (1991). Characterization of the functional preparation of the $70 \mathrm{kD}$ protein of Mycobacterium bovis. Journal of Biological Chemistry 266, 20828-20833.

Pearson, W. R. \& Lipman, D. J. (1988). Improved tools for biological 
sequence comparison. Proceedings of the National Academy of Sciences of the United States of America 85, 2444-2448.

SMITH, D. \& JoHnson, K. (1988). Single step purification of polypeptides expressed in Escherichia coli as fusions with glutathioneS-transferase, Gene 67, 31-40.

Wetzstein, M. \& SchumanN, W. (1990). Nucleotide sequence of a Bacillus subtilis gene homologous to the grp $E$ gene of $E$. coli located immediately upstream of the dnaK gene. Nucleic Acids Research 18, 1289.

Young, D., Lathigra, R., Hendrix, R., Sweetser, D. \& Young,
R. A. (1988). Stress proteins are immune targets in leprosy and tuberculosis. Proceedings of the National Academy of Sciences of the United States of America 85, 4267-4270.

Young, R., Mehra, V., Sweetser, D., Buchanan, T., ClarkeCurtiss, J., Davis, R. \& Bloom, B. (1985). Genes for the major protein antigens of the leprosy parasite Mycobacterium leprae. Nature, London 316, 450-452.

ZhaNG, H., Scoll, R., Browse, J. \& Somerville, C. (1988). Double stranded sequencing as a choice for DNA sequencing. Nucleic Acids Research 16, 1220-1221. 